\title{
Measuring psychological similarity of faces
}

\author{
ALVIN G. GOLDSTEIN and JLNE CHANCE \\ University of Missouri, Columbia, Missouri 65201
}

\begin{abstract}
Recognition memory for unfamiliar other-race faces is poorer than for unfamiliar own-race faces. This observation would be understandable if the within group homogeneity of Japanese faces was greater than white American faces. In a test of this question, both decision time and correctness of same-different judgments offered no evidence to support the hypothesis of differential within group homogeneity.
\end{abstract}

Are white American faces less homogeneous than Japanese faces? The present report describes the first of a series of attempts to measure psychologically whether the variability among structural features comprising Japanese faces is different from the variability found among facial structures of white Americans.

Recognition memory for unfamiliar faces has been demonstrated to be significantly affected by the race ${ }^{1}$ of the observer, and the race of the target face (Chance, Goldstein, \& McBride, 1975; Elliott, Wills, \& Goldstein, 1973; Malpass \& Kravitz, 1969; Cross, Cross, \& Daly, 1971; for review see Ellis, 1975). Briefly summarizing these findings, white subjects' recognition memory performance was best with white faces, poorest with Japanese faces, and intermediate with black faces; black subjects' performance was best with black. poorest with Japanese, and intermediate with white faces.

One explanation of these findings, the differential experience hypothesis (Goldstein, Note 1) is consistent with much of the data and bases its argument on the fact that there are large differences in frequency of exposure to own-race vs. other-race faces. In this view, whites, for example, have infrequent exposure to Japanese faces and therefore might not learn to make the subtle discriminations necessary for precise recognition. The differential experience hypothesis received support from the results of an experiment (Elliott et al., 1973) in which white subjects' recognition memory for unfamiliar Japanese faces was significantly improved as a function of training in a paired associate task which increased their familiarity with (other, nonexperimental) Japanese faces.

Methodological purists could argue, with some justification, that there is no compelling reason to accept the differential experience hypothesis because analysis and control of the stimuli are lacking. Thus, with the data now available, there is no way to decide whether Japanese faces are more difficult to recognize than white faces because they are less often seen by whites or because Japanese faces as a group are more homogeneous than white faces. In other words, samples of white and Japanese (or black) faces used in experiments have been assumed to come from distinct populations in which the mean interstimulus "distance" between individual faces is essentially equivalent. There is no reason to continue to make this assumption, because it is possible to empirically determine the within group homogeneity for each "race" used in experiments. If the assumption of equal range of facial variability was found to be groundless, then Japanese target and distractor faces would be more likely to resemble each other, a situation that generally increases recognition memory errors. A similar argument could be made about black faces. It is of little consequence for the logic of these considerations that blacks and whites (or Japanese) improve their other-race recognition performance after exposure to other-race faces; the need to ascertain the relative homogeneity of the faces of various racial groups is still present.

The hypothesis guiding this research is: if Japanese faces are more alike structurally than white American faces, then white subjects should be expected to take longer and make more errors in deciding whether a pair of Japanese faces are photos of the same individual or photos of two different individuals.

\section{METHOD}

\begin{abstract}
Stimuli
Original black-white photographs of Island Japanese high school graduates were photographed to make 2 by 2 positive black-white slides. This procedure was also used to obtain 2 by 2 slides of American white college seniors. Only men's faces were used. Of the nine pairs of Japanese and nine pairs of white faces used, four pairs in each set were identical photographs and the remaining five pairs were pictures of different people. ${ }^{2}$
\end{abstract}

\section{Procedure}

In several different random orders, pairs of faces were simultaneously projected for $.5 \mathrm{sec}$ by two Carousel projectors, linked together through two Hunter decade timers. Images were adjacent to each other, one always about $50 \%$ larger than the other (a zoom lens controlled image size), and one slightly brighter (either the high- or low-intensity position on the projector's switch was used) than the other. In each of the "same" conditions, one of the pair of identical slides was displayed reversed left to right. All three of these discrepancies between identical slides-size. brightness, left-righ reversal (i.e.. head position)-were introduced to force subjects when making decisions primarily to process the features of the face, and not use cues which are essentially irrelevant to the physiognomic attributes. 
The projectors displayed an image only if the subject was fully depressing both the left and right ("same" and "different") spring-loaded switches simultaneously. When the subject made his decision. he released one of the two switches, thereby stopping an electric clock which had been actuated by the pulse initiating the cycling of the Carousel projectors. Both response switches were clearly labeled and visible to the subject in the semidarkness of the experimental room. Following each presentation of face pairs, opaque slides were automatically cycled into place, throwing the projection screen into darkness until the subject viewed the next pair of faces.

A minimum of 10 practice trials. using nonfacial stimuli, were employed to familiarize each subject with the complete procedure. Decision time and correctness were recorded by the experimenter. seated behind the subject. Snellen visual acuity had been measured before the beginning of the experiment, and prospective subjects (with or without optical correction) who could not correctly report all letters of the 20/30 line (from $20 \mathrm{ft}$ ) were not used. Sixty-three undergraduate subjects were tested in the experiment.

\section{RESULTS AND DISCUSSION}

Analysis of two different measures of accuracy will be reported, latencies of response to correct judgments and number of judgmental errors. Although response latency for both correct and incorrect judgments were available, only latencies for correct responses were analyzed inasmuch as the meaning of incorrect response latencies is ambiguous.

Mean response latency for Japanese faces was $1.07 \mathrm{sec}$, and for white faces was $1.05 \mathrm{sec}$. Mean number of correct responses to Japanese faces was 6.3 as compared to 6.2 for white faces. Analysis of variance of these data support the conclusion, obvious from simply looking at the miniscule differences between mean values, that discrimination of Japanese faces is no more difficult than discrimination of white faces. Separate analyses of "same" response latencies to white $(1.06 \mathrm{sec})$, and Japanese $(1.08 \mathrm{sec})$ faces again reveals almost identical values. Similarly, "different" response latencies to Japanese $(1.09 \mathrm{sec})$ and white $(1.08 \mathrm{sec})$ faces are not reliably different. ${ }^{3}$

These data offer no support for the notion that Japanese faces as a group are more structurally similar than white faces, and therefore are less discriminable than white faces. Needless to say, these conclusions are tentative, dependent as they are on a small sample of faces. If further research supports the present conclusion, one may speculate that differences in recognition memory performance may be more likely attributable to either the storage or retrieval processes than to the process of registration.

\section{REFERENCE NOTE}

1. Goldstein, A. G. Implications of differential face recognition data. Paper presented at the meeting of the Midwestern Psychological Association, Chicago, Illinois, May 1975.

\section{REFERENCES}

Chance, J., Goldstein, A. G., \& McBride, L. Differential experience and recognition memory for faces. Journal of Social Psychology. 1975. 97. 243-253.

Cross, J. F., Cross, J., \& Daly, J. Sex, race, age, and beauty as factors in recognition of faces. Perception \& Psychophysics, 1971, 10, 393-396.

Elliott. E., Wills, E. J., \& Goldstein, A. G. The effects of discrimination training on the recognition of white and oriental faces. Bulletin of the Psychonomic Society, 1973, 2, 71-73.

Ellis, H. D. Recognizing faces. British Journal of Psychology, 1975. 66. 409-426.

Malpass, R. S., \& KRavitz, J. Recognition for faces of own and other "race." Journal of Personality and Social Psychology, 1969, 13, 330-334.

\section{NOTES}

1. "Race" is used in a nontechnical sense, to refer to groups of people who share in common facial features which perceptually distinguish them from other groups.

2. Other stimuli were included in the original experiment, but they are irrelevant to the present discussion. Any effects the additional trials may have exerted on the subjects, and presumably on the data reported here, should be equally dispersed among all subjects and, more importantly, between both Japanese and white stimuli, since systematic randomization of the various stimuli was used in the experiment.

3. While the face stimuli did not differ among themselves, they did differ significantly from the other stimuli in the experiment in both decision times and number of judgments correct.

(Received for publication October 24, 1975.) 